\title{
Representaciones sociales sobre VIH/SIDA en mujeres indígenas miskitas en Puerto Cabezas, Costa Caribe Norte de Nicaragua
}

\author{
Una Mirada Crítica desde el Feminismo Decolonial \\ Social representations about HIV / AIDS in Miskito indigenous women in Puerto Cabezas, North \\ Caribbean Coast of Nicaragua
}

A Critical Look from Decolonial Feminism

Gretta Paiz Malespín ${ }^{1}$

María Sol Pastorino ${ }^{2}$

\section{Resumen}

Esta investigación profundiza en las circunstancias y formas en que mujeres miskitas con VIH sobreviven a esta infección en una región afectada históricamente por la exclusión social y el aislamiento geográfico que la hace particularmente vulnerable a la pandemia. Se propuso ir a los imaginarios sociales y desde allí, discutir en qué condiciones el VIH y el Sida afecta social, económica, espiritual y culturalmente a las mujeres indígenas. Este estudio tiene como marco epistémico el feminismo decolonial desde el cual se analizan condiciones de violencias y desigualdades estructurales, invisibilidad, subordinación, exclusiones y asimetrías de poder. En consecuencia, analiza las condiciones estructurales que hacen que, mujeres miskitas con VIH sean rechazadas y expulsadas de sus espacios y comunidades por un sistema caracterizado por relaciones patriarcales que las obliga a abandonar su hogar y comunidad para apartarse de la discriminación, el estigma y los prejuicios sociales. La investigación, guiada con rigurosidad por las normas y leyes nacionales en materia de promoción, protección y defensa de los derechos humanos ante el VIH y el Sida (Ley 820), reconstruye la realidad tal y como la observan las actoras del estudio, registrando con singularidad, las propias vivencias contadas por mujeres miskitas con VIH. De esta manera, el estudio preserva principios de autonomía, privacidad, consentimiento informado y, confidencialidad de las entrevistas y las/los entrevistados.

1 Candidata Doctoral en Ciencias de la Salud. Asesora de Investigación de la Universidad de las Regiones Autónomas de la Costa Caribe Nicaragüense (URACCAN). Email: grettapaizm@yahoo.com. ORCID: http://orcid.org/oooo-ooo2-6o36-6122.

Doctora en Ciencias Sociales. Centro de Investigaciones y Estudios de la Salud (CIES-UNAN-Managua). Email: lic.msolpastorino@gmail.com. ORCID: https://orcid.org/oooo-0002-5368-8457

Recibido: 08/01/2021 - Aprobado: 05/02/2021 
Palabras clave: Representaciones sociales; VIH/Sida; Mujeres Indígenas Miskitas; Violencia; Feminismo Decolonial.

\section{Abstract}

This research delves into the circumstances and ways in which Miskito women with HIV survive this infection in a region historically affected by social exclusion and geographic isolation that makes it particularly vulnerable to the pandemic. It was proposed to go to the social imaginary and from there, discuss under what conditions HIV and AIDS affect indigenous women socially, economically, spiritually and culturally. This study's epistemic framework is decolonial feminism from which conditions of violence and structural inequalities, invisibility, subordination, exclusions and asymmetries of power are analyzed. Consequently, it analyzes the structural conditions that cause Miskito women with HIV to be rejected and expelled from their spaces and communities by a system characterized by patriarchal relationships that forces them to leave their home and community to move away from discrimination, stigma and abuse. social prejudices. The investigation, rigorously guided by national norms and laws on the promotion, protection and defense of human rights against HIV and AIDS (Law 820), reconstructs the reality as observed by the study actors, registering with singularity, the own experiences told by Miskito women with HIV. In this way, the study preserves principles of autonomy, privacy, informed consent and confidentiality of the interviews and the interviewees.

Keywords: Social representations; HIV AIDS; Indigenous Miskito Women; Violence; Decolonial feminism

\section{Introducción}

A treinta y dos años de la Primera Conferencia Internacional sobre la Promoción de la Salud realizada en Ottawa y el compromiso asumido en la consecución del objetivo "Salud para Todos en el Año 200o" (Carta de Ottawa, 1986, en OPS, 1987), aún persisten los obstáculos que impiden la adopción de políticas que favorezcan la salud para todos. Es un período marcado por nuevas expectativas por erradicar la pobreza extrema y reducir las desigualdades en todas sus dimensiones (Agenda 2030 para el Desarrollo Sostenible en CEPAL, s.f;), y poner fin a la epidemia del VIH y el Sida (Objetivos de Desarrollo Sostenible, en ONUSIDA, s.f y CEPAL, s.f.a).

Han pasado más de tres décadas, mejor dicho, treinta y ocho años después de haberse diagnosticado en Estados Unidos el primer caso positivo de VIH (1981), 
relacionado a infecciones pulmonares poco frecuentes para la época (Roca, 2017). Este es un virus que de acuerdo a las evidencias amenaza la salud pública del planeta (Lamptey, Johnson y Khan, 2006). Se trata de una pandemia que para el 2017 había cobrado más de 35 millones de vida (OMS, noviembre 2017). Parte de la complejidad del VIH y el SIDA es que es agravada por las condiciones sociales, económicas, políticas y culturales de las sociedades que lo enfrentan (Dugassa, 2009).

Dramáticamente, este es un estado que refleja el deterioro de la salud colectiva (Laurell,1986), y "enmascara importantes disparidades" (Naciones Unidas, 2015, p.47). Autores consultados por este estudio, direccionan este llamado deterioro, hacia las grandes desigualdades sociales, que ponen al centro de cualquier debate el mundo de las asimetrías, exclusiones e inequidades (CEPAL, 2016). En tal caso, la discusión tiene como punto de inflexión, el bienestar integral y la calidad de vida que necesita la gente para vivir dignamente (Balladeni, 2015).

\subsection{Salud, mujer y VIH}

\section{El estado de bienestar integral que supone la salud}

De acuerdo a la Organización Mundial de la Salud, la salud, evoca un estado de completo bienestar, físico, mental y social y no solamente, ausencia de afectaciones o enfermedades (OMS, 1946). Por tanto, es una salud que reivindica desarrollo social y económico (OPS/OMS,1997), y equilibrio entre el individuo y su entorno (Dubos, 1967, en Gavidia y Talavera, 2012). Significativamente, la salud comprende paz, vivienda, educación, seguridad social, relaciones sociales, alimentación, empoderamiento de la mujer, ecosistema estable, uso sostenido de los recursos, justicia social, respeto de los derechos humanos, equidad y la ausencia de pobreza (Declaración de Yakarta en OPS/OMS,1997:5).

En el caso de la Costa Caribe de Nicaragua, el derecho constitucional de salud para todos (arto 59 Constitución Política, en La Gaceta, Diario Oficial, 18 de febrero, 2014), presupone acceso a los programas de salud sin discriminación alguna, ni por religión, etnia, raza, sexo, edad, ni por nivel socioeconómico. Esto impone una lógica de igualdad y equidad (CEPAL, 2017:63), que sin duda se hace necesario no sólo para fortalecer los sistemas de salud, sino para enfrentar con respuestas eficaces la desigualdad global de salud (Puyol, 2010:481). Esto refiere, la existencia de condiciones favorables para revertir la pobreza, la extrema pobreza y acceso universal a tratamientos antirretrovirales, sin distinto de ningún tipo. 


\section{2.- Mujer, VIH y el Sida}

\section{Vulnerabilidad y tradiciones culturales}

En el 2005, 17,5 millones de mujeres vivían con el VIH, un millón más que en 2003. La proporción de mujeres afectadas por la epidemia sigue creciendo y el impacto es cada vez mayor sobre las mujeres (ONUSIDA-OMS, 2005), agravado por sus propias condiciones biológicas, culturales y socioeconómicas. Las tradiciones culturales, como la posición dominante de los hombres y la preferencia de éstos por mujeres jóvenes, es un detonante de la vulnerabilidad de la mujer frente al VIH.

La violencia contra la mujer, incluida la violencia sexual, es otro factor en esta transmisión que define el impacto del VIH atribuible a factores biológicos, sociales, económicos y estructurales (Ramjee y Daniel, 2013). En este contexto desfavorable para las mujeres, se hace oportuno definir campañas de prevención con pertinencia, interculturales y horizontales que partan de las propias realidades y experiencias de las mujeres indígenas miskitas y las dificultades que enfrentan para lograr el control sobre sus propias vidas sexuales.

\section{3.- Epidemiología del VIH y el Sida}

En el mundo, 42,9 millones de personas vivían con el Virus de Inmunodeficiencia Humana (VIH) en el primer semestre del 2017, de este total, el 20,3 millones son mujeres mayores de 15 años, y, 1,2 millones de personas murieron a causa de enfermedades relacionadas con el sida (ONUSIDA, 2017b), "a pesar de la existencia de terapias anti-retrovirales que intervienen en la trayectoria de la enfermedad y pueden prolongar la vida”. (Zambrano, 2013).

En Nicaragua, el primer caso de VIH fue diagnosticado oficialmente en 1987 y en 1988 en la Costa Caribe (CORESIDA/MINSA, 2009). Su impacto se reconoce de la siguiente manera:

La epidemia del VIH y el Sida es una realidad en Nicaragua, y cuya trascendencia se expresa no solamente en la salud de las personas que viven con el VIH, sino en la forma como afecta su núcleo familiar, comunitario y social, con un fuerte impacto socioeconómico en el desarrollo humano de la población y del país. (MINSA/ CONISIDA, 2006:11).

Para el 2017, Nicaragua registraba 4 mil 260 pacientes con VIH que accedían a sus terapias antirretrovirales en un contexto en el que la epidemia estaba concentrada 
en hombres teniendo sexo con hombres, transexuales, trabajadoras sexuales, población adolescente y joven en general (MINSA, 30 noviembre 2017). En el caso particular del Caribe Norte de Nicaragua, este mismo año fueron reportados 65 los nuevos casos y 21 los fallecidos. El año anterior, (2016), habían sido 109 los nuevos casos y 30 los fallecidos en la etapa Sida (SILAIS/BILWI, 2016-2017).

Y pese a que son datos bastante generales, en la Región, el VIH y el Sida es un problema creciente en abierta alusión a un problema global de salud y/o ausencia de bienestar integral, y, la ausencia de oportunidades en materia de salud (Whitehead,1991) como condicionante de la ausencia de equidad, que salud implica que, idealmente, todas y todos consigan acceder a oportunidades justas en salud y, que al lograrlo, quede evidenciado, que las barreras pudieron evitarse (Copenhagen, 1986, en Whitehead,1991:7).

\section{4.- Contexto del estudio}

Tiene como contexto, condicionantes adversas y desfavorables a las mujeres en un territorio en donde se sospecha que tanto el VIH como el sida han incrementado en un marco de violencia y en un escenario justificado por un mimetismo, hermetismo e invisibilización de un problema grave de salud pública. Es un contexto justificado por un sistema de prejuicios sociales que incentivan patrones de discriminación y exclusión social que repercute en su calidad de vida, su salud, su vida cultural, familiar y espiritual.

Con este planteamiento esta investigación hace énfasis en los siguientes aspectos relevantes:

\subsubsection{Un estudio con perspectiva crítica feminista decolonial}

Es un estudio que utiliza la perspectiva crítica feminista decolonial para levantar y recoger la voz de la mujer con VIH, reconocer su experiencia y comprender sus procesos de significación que ha construido a partir de su rol de género (Flores, 2014:47). Se utiliza la epistemología del feminismo decolonial como la manera de visibilizar su situación y realidad (Blazquez, Flores y Ríos, 2012) en un estudio que asume una postura crítica frente a la complejidad que viven mujeres miskitas con VIH en la Costa Norte de Nicaragua marcadas por una "herida colonial" que las humilla y las hace sentir inferior" (Mignolo, 2016).

Coherentemente con la realidad estudiada, la opción decolonial hace posible desde sus propios instrumentos de análisis, sumergirnos en el mundo vivido por 
las mujeres indígenas miskitas del estudio. Se trata de un abordaje decolonial en la que intervienen otros cuerpos, otras mentes, otras memorias y se construyen significativamente otros saberes en aras de descolonizar "el sentir, el hacer y el creer" (Mignolo, 2014) desde una "solidaridad sin frontera" (Gargallo, 2012).

\subsubsection{Propósitos y Relevancia del Estudio}

Por todo lo planteado, este estudio tiene como propósito recuperar los imaginarios sociales y culturales que permean la historia de vida de mujeres indígenas con VIH y que históricamente han permanecido invisibilizados y ausentes de los distintos espacios que se configuran en la comunidad y la familia. El esfuerzo estuvo dirigido a aprehender el sistema de representaciones sociales que posee la sociedad en Bilwi respecto al VIH y que en consecuencia agrava la situación de salud de las mujeres que lo viven (ONUSIDA-OMS, 2005).

Asimismo, problematiza la manera en que se representa social y culturalmente el tema del VIH, en abierta alusión a patrones configurativos de las asimetrías, la invisibilización y el silenciamiento de los grupos subalternos (Chakravorty y Giraldo, 2003).

\subsubsection{Características del Municipio de Puerto Cabezas}

Considerada la columna vertebral de la Autonomía Regional, dado que es la sede de las principales instituciones rectoras del Régimen Autonómico, el Gobierno y Consejo Regional Autónomo, así como las principales instituciones del Estado Nicaragüense. "Esta ciudad es una de las sedes en donde actualmente opera el régimen político de la Autonomía" (Barbeyto, 2014:16). La población se caracteriza por ser multiétinica y pluricultural, el $29 \%$ vive en el área urbana y el $71 \%$ en el área rural, estos últimos dispersos en 470 comunidades (Sequeira, Perera, Cruz y Quino, 2008).

Por su ubicación geopolítica, es una ciudad portuaria concurrida por personas de todas partes del país y de otros municipios. Entre sus habitantes, vamos a encontrar a gente procedentes del Río Coco, Litoral Norte, Litoral Sur, Llano Norte, Llano Sur, entre otros territorios de la región (Escobar, 2009; Marley, 2010). Waspam se encuentra aproximadamente a 150 kilómetros de Bilwi. Es un municipio fronterizo con Honduras, uno de los países centroamericanos con alto índice de casos. Su condición de país fronterizo influye en que las personas emigren de un lugar a otro frecuentemente. 


\subsubsection{El VIH en la ruta de las representaciones sociales}

Asumiendo que toda representación social es representación de algo y de alguien (Jodelet, 1985; Moscovici, 1961), este estudio escudriña en el entretejido de los propios imaginarios de mujeres y hombres en Puerto Cabezas, la representación que se produce "con el mundo y con las cosas" (Matus, 2016). En tal sentido, evoca a aprehender las representaciones que la sociedad configura a manera de construcciones sociales (Parker, 1989, citado en Rodríguez, 2003).

Para este estudio, las representaciones aquí discutidas apuntan a un proceso desde el cual se recoge, estructura y, se intercambian representaciones culturales y prácticas significativas entre los integrantes de una cultura (Hall, 2010:447; FloresPalacios 2014:9). Dicho de otro modo, las representaciones interconectan procesos, contenidos, condiciones y contextos en las que estas se originan y por tanto, deben explicitarse.

\section{II.Desarrollo}

\section{II.1 Las violencias contra mujeres miskitas con VIH}

Entre el 2007 al 2010, y los años que siguieron en la Costa Caribe Norte de Nicaragua fueron períodos marcados por avances y desafíos importantes en la respuesta. En el período destacaron estrategias dirigidas a la prevención, atención y tratamiento impulsados por la Comisión de Lucha Contra el Sida del Centro Clínico Bilwi, en consorcio con Horizont 3000 y el Ministerio de Salud. Sin embargo y pese a los grandes esfuerzos realizados en la región por revertir los niveles de prevalencia del VIH y el SIDA en la Costa Caribe de Nicaragua, con la llegada de los años 2011 y 2012, los proyectos orientados a la prevención y atención del VIH y el Sida fueron cerrando.

El financiamiento de Horizont 3000 se agotó, y con ello, finalizaron todas las acciones dirigidas a la respuesta. En un escenario desalentador, disminuyeron las pruebas gratuitas para detectar el VIH y el Sida así como los antirretrovirales. Esto llevó a personas con VIH atendidos tanto por la Comisión de Lucha Contra el Sida del Centro Clínico Bilwi y el Policlínico a abandonar el tratamiento, con paraderos desconocidos hasta el día de hoy.

De allí, la importancia de indagar y conocer las condiciones en que los programas y estrategias que un día fueron tan buenos porque llenaron de esperanza a personas con VIH (hombres y mujeres), simplemente desaparecieron, y en cambio, 
lo que existe hoy son altos niveles de preocupación e incertidumbre frente a uno de los problemas de salud pública más sentidos en el municipio de Puerto Cabezas, seguido de la malaria.

Siguiendo las evidencias establecidas, este estudio define y es guiado por las siguientes preguntas:

1. ¿Por qué el proceso de producción de las estrategias dirigidas a la respuesta del VIH y el SIDA como factor determinante ha sido insuficiente para responder a la situación del VIH y el SIDA en pueblos indígenas de la Costa Caribe Norte de Nicaragua?

2. ¿Por qué si han habido distintas estrategias dirigidas a la prevención, atención y tratamiento del VIH y el Sida, hoy, hombres responsables de haber infectado a mujeres participantes de este estudio, siguen infectando a otras mujeres y re-infectando a su propia pareja?

3. ¿Porqué hablar hoy de VIH se ha convertido en un problema, mientras las instituciones encargadas de liderar el tema lo manejan con total hermetismo?

4. ¿De qué manera el sistema de prejuicios, estereotipos, estigma y discriminación agravan la situación de la mujer miskita con VIH y el SIDA en el municipio de Puerto Cabezas, Caribe Norte de Nicaragua y cómo este sistema se asocia a formas de violencia estructurales?

5. ¿Cuáles son las implicancias de vivir con VIH y SIDA? (personales, individuales, familiares, comunitarias, laborales y de pareja)

6. ¿Por qué el sistema de representaciones que se configuran socialmente agravan la situación de mujeres miskitas con VIH y el SIDA en el municipio de Puerto Cabezas, Caribe Norte de Nicaragua?

7. ¿Cuáles con las representaciones sociales asociados al VIH y al SIDA en mujeres miskitas en Puerto Cabezas? Y, ¿Cuáles son los significados colectivos del VIH y el SIDA que dominan en la Costa Caribe Norte de Nicaragua?

Congruente con lo planteado, este estudio discute desde las entrañas del feminismo decolonial el sistema de representaciones en que se instaura y configuran las violencias, abarcadoras de exclusiones, inequidades, prejuicios, discriminación, estigma y desigualdades en contra de mujeres con VIH, perjudicial a su salud (Sen y Östlin, 2007), y, porque sin duda, el VIH y el Sida causa exclusión social en la medida que empeora más la enfermedad (Deossa, 2009). 
Por todo lo anterior, el presente estudio -articulado desde la perspectiva paradigmática de Stuart Hall (1932-2014) y los estudios culturales--, representa un intento coherente y necesario por identificar las representaciones y los imaginarios sociales que se configuran en el seno de una sociedad con respecto a mujeres con $\mathrm{VIH}$, analizar los procesos de producción de dichas representaciones.

\section{II.2 Desde Stuar Hall hasta la Costa Caribe Norte de Nicaragua}

El debate sostenido en este marco conceptual se fundamenta y estructura en tres partes:

\section{La primera parte:}

Aborda el concepto de representaciones discutido por distintas tradiciones teóricas con especial énfasis desde Stuart Hall y los Estudios Culturales, consiguiendo analizar desde el circuito de la cultura propuesto por los estudios culturales, la manera en que se configuran las representaciones sociales desde el propio proceso de producción.

Este acápite busca identificar las estrategias, los programas y las acciones dirigidas a la prevención, atención y tratamiento del VIH y el Sida en contextos de Pueblos Indígenas, particularmente en territorios de la Costa Caribe de Nicaragua, y de esta manera, poder dar cuenta del tipo de intervenciones implementadas por las instituciones regionales que lideran el tema de salud en el Caribe Norte del país, así como los cambios que explican la situación actual del VIH y el SIDA en el municipio de Puerto Cabezas.

\section{La segunda parte:}

Se propone evidenciar las principales manifestaciones de la violencia en contra de mujeres miskitas con VIH en el municipio de Puerto Cabezas en la Costa Caribe Norte de Nicaragua, en un escenario marcado por un mimetismo, hermetismo e invisibilización de un problema que las transgrede, afecta y repercute gravemente en su calidad de vida, su salud, su vida cultural y espiritual.

El debate sugerido, se articula desde la mirada crítica y epistémica del feminismo decolonial cuyo enfoque y postura lleva a develar los niveles de violencia, de género, estructural y en nombre de la tradición que enfrentan las mujeres miskitus con VIH en la Costa Caribe Norte de Nicaragua, una región urgida de estrategias dirigidas 
a reducir el estigma, la discriminación, los estereotipos y la descolonización de la violencia contra las mujeres.

Desde la lógica de Cabnal, este estudio inflexiona el tema de las opresiones, y cómo éstas atraviesan el cuerpo de las mujeres indígenas con VIH y que lamentablemente tiene expresión en el racismo, el colonialismo, y las formas de hegemonía de pensamientos dominantes. De manera, que es un cuerpo en disputa y que desde la lógica del sistema patriarcal no tiene propiedades y tampoco tiene enunciados de la apropiación de este cuerpo, que dramáticamente ha sido expropiado y por ende pertenece al sistema patriarcal.

\section{La tercera parte:}

Analiza los elementos configurativos de una posible relación existente entre las desigualdades estructurales, la pobreza, la extrema pobreza, el patriarcado y las opresiones al incremento del VIH y el Sida en poblaciones indígenas y el deterioro en su calidad de vida. Este representa un eventual escenario guiado desde la postura del feminismo comunitario en Abya Yala y cuya crítica radica en el llamado a interpretar la configuración del sistema patriarcal en esta territorialidad ancestral de los pueblos llamada Abya Yala ("la sangre que corre libre").

En este debate, destaca la mirada de Arelys Barbeyto (2014), antropóloga y doctora en ciencias sociales, especialista en temas relacionados a la violencia anclada en un sistema de género; Lorena Cabnal (2016), feminista comunitaria y líder indígena guatemalteca, Nuria Varela (2017), periodista y escritora española especialista en "micromachismos" como extensión de las violencias simbólicas, culturales y desigualdades estructurales, y Johan Galtung, autor que define la violencia cultural como "cualquier aspecto de una cultura que puede ser utilizada para legitimar la violencia en su forma directa o estructural”. (Galtung, 2016:147). Entre otras y otros destacados autores.

Básicamente este estudio se propone evidenciar la manera en que la gente, recogen, estructuran y comparten las representaciones culturales y prácticas significativas en el contexto de la realidad social (Flores-Palacios, 2014:9) de la gente de la Costa Caribe nicaragüense. En este sentido, los estudios culturales importan porque representan una formación cultural vigente (Caloca, 2015:18) y pertinente para analizar desde una perspectiva contemporánea (Sánchez, 2014:6) el fenómeno de las representaciones en un contexto donde la diversidad cultural tiene como anclaje grupos de individuos, indígenas y afrodescendientes. 


\section{III.3 Violencias, prejuicios y estereotipos}

Mujeres miskitas con VIH consultadas en ocasión de este estudio, aseguran que viven una violencia que las reduce, las discrimina y las marginaliza. Dramáticamente, son mujeres que están afrontando en condiciones desiguales, tanto fuera como dentro de sus propias comunidades formas de discriminación y marginación gravitadas por su sexo, género, raza y color de la piel, etnicidad, edad, situación de pobreza y los prejuicios sociales (Naciones Unidas, 2015; CIDH, 2017; Miguens, 1970; Rose, 1970), este último en alusión al sesgo y entorpecimiento con el que se percibe una realidad incompleta (Gamarnik, s.f; Adichie, 2009).

En Consecuencia, son prejuicios que es la práctica homogeniza, banaliza, simplifica, generaliza la información, ignora los verdaderos conflictos y oculta la verdadera historia. De allí, la relevancia de transcender desde la epistemología feminista decolonial, al debate de las condiciones estructurales en que mujeres indígenas en el Caribe Norte de Nicaragua, han venido construyendo y configurando sus voces individuales y colectivas respecto a la violencia, la discriminación, el estigma y la exclusión. En todo caso, es una discusión que inflexiona desde las propias realidades y experiencias de las mujeres (Harding, 1993; Harding, 1987; Blazquez, Flores y Ríos, 2012).

\section{II.4 Una región con exclusiones sociales históricas}

La Costa Caribe de Nicaragua registra una historia de exclusión social y aislamiento geográfica que hace este territorio especialmente vulnerable a factores como la pobreza, alta prevalencia de infecciones de transmisión sexual, el inicio de la vida sexual activa a temprana edad, prácticas sexuales de riesgo y debilidades en el sistema de salud (UNICEF, 2007). Esto explica en parte, el comportamiento del VIH y el SIDA en esta parte del territorio nacional.

Pese a esfuerzos de instituciones y organizaciones que por años se han sumado a la respuesta en la región, el tema del VIH y el SIDA hoy, está lacerando las vidas de mujeres pertenecientes a pueblos indígenas, infectadas por sus propias parejas (Marley, 2010). De allí la importancia de investigar para intervenir en una realidad que modifica la situación de pobreza, extrema pobreza y serias repercusiones en la salud (Zambrano et al, 2013).

De acuerdo al Ministerio de Salud de Nicaragua, la estrategia de atención a pacientes con VIH está dirigido a asegurar el acceso a programas de prevención y atención integral (MINSA, 30 noviembre, 2017). Lamentablemente, este reporte 
oficial del MINSA, no coincide con información confiable recabada en el territorio donde se registra abandono del tratamiento e incremento del VIH en poblaciones indígenas, principalmente en mujeres.

\section{II5. Aprehendiendo las representaciones sociales}

\section{La "Grounded Theory" como estrategia metodológica}

La investigación se funda en la metodología cualitativa para asegurar "reconstruir" la realidad tal y como la observan las y los actores. Es un estudio guiado en su estrategia metodológica desde el planteamiento realizado por Taylor y Bogdan (1992), cuyo énfasis lleva a esta investigación a incorporar como rasgos propios la condición inductiva, holística, interactiva, reflexiva y naturalista. En consecuencia, es un estudio que tiene como propósito aprehender la realidad social (HernándezArteaga, 2012) desde las propias perspectiva de quienes la originan y la viven (Hernández-Arteaga, 2012, p.62).

Desde la estrategia de investigación cualitativa previamente establecida se utilizó como herramienta central de recolección de los datos, la entrevista de profundidad (Bryman,1988) aplicadas, entre otros actores relevantes en el territorio, a mujeres con VIH. El estudio incluyó un rastreo bibliográfico y la examinación de una diversidad de fuentes documentales (Mireles-Vargas, 2015).

También incorpora los grupos de discusión y observación del contexto, guiados por la tradición Taylor y Bogdan, en una búsqueda por aprehender las formas en que las y los participantes asignan significados a sus actividades y problemas (Silverman, 2006) capturando un contexto construido de forma diferente (Silverman, 1993).

Es un estudio guiado por la Teoría Fundamentada como estrategia metodológica y para aprehender la realidad social que tienen los distintos actores involucrados en este estudio (Damas, 2019; Strauss y Corbin, 1990). Significativamente, posibilitó, interpelar la realidad social que viven mujeres indígenas miskitas con VIH (Franco y Morillo, 2016), sin hipótesis ni ideas preconcebidas (Cuñat, s.f).

\section{II.6 Un muestreo teórico e intensionado}

\section{Respeto a la integridad, la dignidad y la confidencialidad}

El muestreo es intencionado y de máxima homogeneidad (Patton, 1990), el cual aseguró un grupo de mujeres con VIH con iguales características. El estudio es 
coherente con los resguardos éticos establecidos en la Ley de Promoción, Protección y Defensa de los Derechos Humanos ante el VIH y Sida, para su prevención y atención (Ley 820 en Asamblea Nacional, 2012).

Su visión holística e intercultural del respeto, cuida y preserva principios de autonomía, privacidad, integridad, libertad, consentimiento previo e informado, confidencialidad, dignidad humana y autonomía de las víctimas del VIH en el territorio (Código de Nuremberg 1947 en Centro Interdisciplinario de Estudios de Bioética, s.f); Constitución Política de Nicaragua, en La Gaceta, Diario Oficial, 18 de Febrero del 2014; Ley 28 (Ley de Autonomía); Modelo de Salud Intercultural (MASIRRAN); Convenio 169; Pauta 9-10, CIOMS, 2016; Lema, Toledo, Carracedo y Rodríguez, 2013:25; Declaración de Helsinki, 1964).

\section{Conclusiones}

La primer gran conclusión a la que llega parte del debate incorporado en esta tesis doctoral que se articula desde el análisis de tres grandes aspectos que darán salida a artículos distintos, alude a representaciones sociales configurativas de discriminación, estigma, exclusión, olvido y en consecuencia, de violencias y desigualdades estructurales en contra de mujeres miskitas con VIH en Puerto Cabezas.

Este es un estudio que analiza y desnuda el modo en que la pobreza y la extrema pobreza, expone a las mujeres indígenas miskitas con VIH a formas de violencias que las estigmatiza, las excluye, las cosifica y las discrimina. Sus relatos abren heridas coloniales que permean las condiciones en que les toca vivir en el contexto de una sociedad y comunidad altamente deshumanizada y prejuiciosa.

Que pese a los avances registrados por el sistema de salud en materia de respuesta frente al VIH y el Sida, las evidencias desvelan la persistente desigualdad a la que están expuestas. Esto sin duda, representa un desafío para el Estado de Nicaragua, los Gobiernos Regionales Autónomos, los municipales y los territoriales en la Costa Caribe, por priorizar y construir sociedades menos desiguales, más justas e inclusivas, igualitarias, democráticas e interculturales. Respetuosas de sus derechos individuales y colectivos como pueblos indígenas miskitos en materia de salud. 


\section{Lista de referencia}

Asociación Médica Mundial (AMM). (1964). Declaración de Helsinki de la AMM. Principios Éticos para las investigaciones médicas en seres humanos. Recuperado en: https://www.wma.net/es/policies-post/declaracion-de-helsinki-de-laamm-principios-eticos-para-las-investigaciones-medicas-en-seres-humanos/

Adichie, Chimamade. (2009). El peligro de la historia única. Recuperado en: https:// www.ted.com/talks/chimamanda_ngozi_adichie_the_danger_of_a_single_story?language $=\mathrm{es}$

Asamblea Nacional (2012). Ley 820: Ley de Promoción, Protección y Defensa de los Derechos Humanos ante el VIH y Sida, para su prevención y atención. Recuperado en: http://legislacion.asamblea.gob.ni/SILEG/Iniciativas.nsf/o/e254dbofc 9127ecco62577ddoo59242d/\%24FILE/Ley\%20No.\%20820\%20VIH\%20y\%20 Sida\%2ofinal.pdf

Bryman, Alan. (1988). Quantity y Qualitity in Social Research, Ed. Routledge, Londres. Traducción María José Llanos Pozzi. Universidad de Buenos Aires, Argentina.

Blazquez, N., Flores F., y Ríos, M. (Coord.) (2012). Investigación Feminista. Epistemología, metodología y representaciones sociales. México: UNAM. Colección Debate y Reflexión.

Barbeyto, A. (2014). Mujeres Miskitas, Ciudadanía y Justicia: Violencia Doméstica en Krukira W. Tesis Doctoral en Ciencias Sociales. FLACSO, Guatemala.

Balladeni, P. (2014). Los desafíos de la Salud Global. OMS/OPS. Recuperado en: http://www.paho.org/arg/images/gallery/Salud\%2oGlobal\%20U\%20San\%20 Andres\%202014.pptx?ua=1

C169 (1989). Convenio 169 sobre pueblos indígenas y tribales Organización Internacional del Trabajo.

Chakravorty, S. y Giraldo, S. (2003). Nota Introductoria. En: Chakravorty, S. y Giraldo, S. (2003). ¿Puede Hablar el Subalterno?. Revista Colombiana de Antropología, (39), 297-364.

Consejo Regional del Sida (CORESIDA) y Secretaria de Salud RAAS (MINSA) (2009). Vigilancia Comunitaria de las Infecciones de Transmisión Sexual. Región Autónoma del Atlántico Sur. Recuperado en: http://en.unicef.org.ni/media/ publicaciones/archivos/Vigilancia_Comunitaria_ITS-RAAS.pdf 
Caloca Lafont, Eloy.(2015). Significados, identidades y estudios culturales: Una introducción al pensamiento de Stuart Hall. Razón y Palabra, 19 (2), 1-32.

CEPAL (Comisión Económica para América Latina y el Caribe) (2016). Panorama Social de América Latina. Recuperado en: https://www.cepal.org/es/ publicaciones/41598-panorama-social-americalatina-2016

Cabnal, L. (2016). El Feminismo Comunitario. Entrevista a Lorena Cabnal, feminista comunitaria indígena maya-xinka en el marco del seminario "Los velos de la violencia: Reflexiones y experiencias étnicas y de género en Chile y América Latina". Recuperado en: https://www.youtube.com/watch?v=87DqYYIiSro

CIOMS (2016). Pautas éticas internacionales para la investigación relacionada con la salud con seres humanos. Consejo de Organizaciones Internacionales de las Ciencias médicas en colaboración con la Organización Mundial de la Salud (OMS).

CEPAL (Comisión Económica para América Latina y el Caribe) (2017). Panorama Social de América Latina. Recuperado en: https://www.cepal.org/es/ publicaciones/42716-panorama-social-america-latina-2017

Centro Interdisciplinario de Estudios de Bioética (S.F). Código de Nuremberg. Tribunal Internacional de Nuremberg, 1947. Universidad de Chile. Recuperado en: http://www.uchile.cl/portal/investigacion/centro-interdisciplinario-de-estudios-en-bioetica/documentos/76028/ el-codigo-de-nuremberg

CEPAL (S.F). Acerca de la Agenda 2030 para el Desarrollo Sostenible. Recuperado en:https://www.cepal.org/es/temas/agenda-2030-desarrollo-sostenible/ acerca-la-agenda-203o-desarrollo-sostenible

CEPAL (S.Fa). Objetivos de Desarrollo Sostenible (ODS). Recuperado en:https:// www.cepal.org/es/temas/agenda-2030-desarrollo-sostenible/ objetivos-desarrollo-sostenible-ods

Cuñat, R. (S.F). Aplicación de la Teoría Fundamentada (Grounded Theory) al estudio del proceso de creación de empresas.

Deossa, G. (2009). Inequidades en salud relacionadas con el VIH/SIDA. Perspectivas en Nutrición Humana. 11(1), p.93-97, Universidad de Antioquia, Medellín Colombia. 
Dugassa, B. (2009). Women's rights and women's health during HIV/AIDS epidemics: the experience of women in sub-Saharan Africa. Health Care Women Int., 30(8):690-706. doi: 10.1080/07399330903018377.

Damas, L. (2019). Teoría Fundamentada en Datos como alternativa investigativa para significar el cuidado en la enfermería cubana. Revista Cubana Enfermería (Internet), 35 (3)

Escobar, G. (2009). "Discriminación Social hacia Mujeres viviendo con el VIH y Sida: Una mirada desde la cosmovisión Indígena miskitu". Tésis de Maestría en la Gestión para la Atención y Prevención del VIH y Sida con Enfoque Intercultural. Universidad de las Regiones Autónomas de la Costa Caribe Nicaragüense (URACCAN).

Flores, F. (2014). Vulnerabilidad y Representación Social de Género en Mujeres de una comunidad migrante. Península, IX (2), 41-58.

Flores-Palacios, F. (2014). Presentación, pp. 9-16. En: Flores-Palacios, F. (Coord.) (2014). Representaciones sociales y contextos de investigación con perspectiva de género. Universidad Nacional Autónoma de México.

Franco, Y., y Morillo, J. (2016). Glasser y Strauss: Construyendo una teoría sobre apropiación de la gaita zuliana. Revista de Ciencias Sociales (RCS), XXII, (4), 115-129.

Gargallo, F. (2012). Feminismos desde Abya Yala. Entrevista a Francesca Gargallo. A propósito del Libro Feminismos desde Abya Yala. Ideas y proposiciones de las mujeres de 607 pueblos en nuestra América. https://www.youtube.com/ watch?v=xjgHfSrLnpU

Gavidia, V y Talavera M. (2012). La construcción del concepto de salud. Didáctica de las Ciencias Experimentales y Sociales. 26, 161-175. Doi:10.7203/DCES.26.1935

Galtung, J. (2016). La violencia: cultural, estructural y directa. Cuadernos de estrategia, 183.

Gamarnik, C. (S.F). Estereotipos Sociales y Medios de Comunicación: Un Círculo Vicioso. Question, 1 (23). Recuperado de: http://perio.unlp.edu.ar/ojs/index. $\mathrm{php} /$ question/article/view/826/727 
Harding, S. (1987). Feminism and Methodology. Social Science Issues. Indiana University Press.

Harding, S. (1993). Ciencia y Feminismo. Ediciones Morata.

Hall, S. (2010). El espectáculo del "Otro", pp.419-445. En: Restrepo, Walsh y Vich (Edit.) (2010). Sin Garantías: Trayectorias y problemáticas en estudios culturales. Instituto de estudios sociales y culturales Pensar, Universidad Javeriana, Instituto de Estudios Peruanos, Universidad Andina Simón Bolivar, sede Ecuador, Envión Editores.

Hernández-Arteaga, I. (2012). Investigación cualitativa: una metodología en marcha sobre el hecho social. Rastros Rostros, 14 (27), 57-68.

Jodelet, D. (1985). La representación social: fenómenos, concepto y teoría, pp.469-494. En: Moscovici, S. (1985). Psicología Social II, Buenos Aires, Paidós.

Rose, A. (1970). La ignorancia acerca de otros grupos humanos, causa de prejuicios, pp.29-34. En: Rose, A. (1970). El origen de los prejuicios. Buenos Aires. Hvmanitas, Buenos Aires. Argentina.

Rodríguez, T. (2003). El debate de las representaciones sociales en la psicología social. Relaciones 93, vol. XXIV. Universidad de Guadalajara, México.

Ramjee, G., y Daniel, B. (2013). Women and HIV in Sub-Saharan Africa. AIDS Research and Therapy. Recuperado en: http://www.aidsrestherapy.com/ content/10/1/30

Roca, M. (2017). "Factores relacionados con el insuficiente control de la infección por el VIH en la Cohorte Española VACH”. Universitad Jaume I.

Laurell, A. (1986). El estudio social del proceso salud-enfermedad en América Latina. Cuadernos Médico Sociales, 37. Recuperado en: http://www.amr.org. $\mathrm{ar} / \mathrm{amr} / \mathrm{wp}$-content/uploads/2015/10/n37a138.pdf

Lamptey, P., Johnson, J., y Khan, M. (2006). El desafío mundial del VIH y el SIDA. Population Bulletin, 61 (1). Washington, DC: Population Reference Bureau.

Lema, S., Toledo, S., Carracedo, M., Rodríguez, H. (2013). La ética de la investigación en seres humanos en debate. Revista Médica Uruguaya, 29 (4), 242-247. 
La Gaceta, Diario Oficial (18 de Febrero del 2014). Constitución Política de Nicaragua con sus Reformas incorporadas.

Silverman, D. (1993). Beginning Research. Interpreting Qualitative Data. Methods for anlysing Talk, Text and Interaction. Sage Publications.

Strauss, A., y Corbin, J. (1998). Bases de la investigación cualitativa. Técnicas y procedimientos para desarrollar la teoría fundamentada. Sage, Publicaciones.

Silverman, D. (2006). Interpreting Qualitative Data. Third Edition. Sage Publications.

Sen G., Östlin P., y George A. (2007). La inequidad de género en la salud: desigual, injusta, ineficaz e ineficiente. Por qué existe y cómo podemos cambiarla. Organización Panamericana para la Salud. Recuperado en: http://www.paho.org/hq/index. php?option=com_docman\&task=doc_details\&gid=1982\&Itemid=270\&lan $\mathrm{g}=\mathrm{en}$

Sequeira, G., Perera, JF., Cruz, M., y Quino, N. (2008). "Evaluación de Conocimientos, Actitudes y Prácticas sobre Salud Reproductiva y VIH/SIDA en estudiantes de la universidad URACCAN, Recinto Bilwi, durante el período de agosto 2006-2007". Universidad de las Regiones Autónomas de la Costa Caribe Nicaragüense (URACCAN).

Sánchez, C. (2014). Reflexiones en torno al concepto de representaciones y su uso en la historia cultural. Questión, Revista especializada en Periodismo y Comunicación, pp.1-15.

SILAIS BILWI (2016/2017). Enfermedades infecciosas. Región Autónoma de la Costa Caribe Norte (RACCN). SILAIS Bilwi. Recuperado en: http://mapasalud. minsa.gob.ni/mapa-de-padecimientos-de-salud-silais-bilwi-raccn/

Moscovici, S. (1961). El Psicoanálisis, su imagen y su público. Buenos Aires: Huemul.

Miguens, J. (1970). Prólogo, p.9-18. En: Rose, A. (1970). El origen de los prejuicios. Editorial Hvmanitas, Buenos Aires, Argentina.

MINSA/CONISIDA(2006). Plan Estratégico Nacional de ITS, VIH y Sida. Nicaragua 2006-2010. Comisión Nicaragüense del SIDA. 
Marley, A. (2010). Análisis Intercultural y Cosmogónico del VIH/Sida en las experiencias de los Miembros del Club de Vidas Futuras de Bilwi en la RAAN. Tésis inédita para optar al grado de Máster en Salud Intercultural. Universidad de las Regiones Autónomas de la Costa Caribe Nicaragüense (URACCAN).

Mignolo, D. W. (2014). El concepto de (de) colonialidad y América Latina. Conferencia Walter D. Mignolo en FHCE/UDELAR. Recuperado en: https://www.youtube. com/watch?v=xjgHfSrLnpU

Mireles-Vargas, Olivia. (2015). Metodología de la investigación: operaciones para develar representaciones sociales.

Matus, P. (2016). Representaciones e imaginarios del medio ambiente en la publicidad de Empresas en Chile. Una aproximación desde avisos en diarios. (Tesis Doctoral, Pontificia Universidad Católica de Chile).

Mignolo, W. (2016). La opción decolonial como proyecto, una entrevista con Walter Mignolo. Recuperado en: https://www.youtube.com/watch?v=xjgHfSrLnpU

Ministerio de Salud. (MINSA). (30 de noviembre, 2017). Analizan resultados del estudio de poblaciones claves para fortalecer respuesta ante el vih. Recuperado en: http://www.minsa.gob.ni/index.php/noticias-2017/3805-analizanresultados-del-estudio-de-poblaciones-claves-para-fortalecer-respuestaante-el-vih

Naciones Unidas (2015). Informe de la Relatora Especial sobre los derechos de los pueblos indígenas. Victoria Tauli Corpuz.

Organización Mundial de la Salud (OMS) (1946). Preámbulo de la Constitución de la Asamblea Mundial de la Salud, adoptada por la Conferencia Sanitaria Internacional, Nueva York, 19-22 de junio. Recuperado en: http://www.who. int/governance/eb/who_constitution_sp.pdf

Organización Panamericana de la Salud (OPS) (1987). Carta de Ottawa para la promoción de la salud. Primera Conferencia Internacional sobre la Promoción de la Salud. Ottawa, Canadá, 21 de Noviembre de 1986. Recuperado en: http:// www1.paho.org/spanish/HPP/OttawaCharterSp.pdf

OPS/OMS (1997). Declaración de Yakarta sobre la Promoción de la Salud en el Siglo XXI. Cuarta Conferencia Internacional sobre la Promoción de la Salud, 2125 de julio, 1997. Recuperado en: http://www.who.int/healthpromotion/ conferences/previous/jakarta/en/hpr_jakarta_declaration_sp.pdf 
ONUSIDA/OMS (2005). Situación de la epidemia de SIDA, diciembre de 2005: y ONUSIDA, Informe sobre le epidemia mundial de SIDA, $4^{\circ}$ informe mundial (Ginebra: ONUSIDA, 2004).

Organización Mundial de la Salud. (OMS) (noviembre 2017). 10 datos sobre el VIH/ sida. Recuperado en: http://www.who.int/mediacentre/factsheets/fs36o/es/

ONUSIDA (2017b). Estadísticas Mundiales sobre el VIH. Recuperado en: http://www. unaids.org/es/resources/fact-sheet

ONUSIDA (S.F). El SIDA y los Objetivos de Desarrollo sostenible. Recuperado en: https://www.unaids.org/es/AIDS_SDGs

Pattón, M. (1990). Qualitative evaluation and research methods. (pp. 169-186). Beverly Hills, CA: Sage.

Puyol Á. (2010). Salud y justicia global. Isegoría. Revista de Filosofía Moral y Política. 43, 479-502.

Taylor, S. y Bogdan, R. (1992). Introducción a los métodos cualitativos de investigación. La búsqueda de los significados. Edición Paidós, España.

UNICEF (2007). La epidemia del VIH en Nicaragua. Recuperado en: https://www. unicef.org/lac/Hoja.pdf

Varela, N. (2017). Cansadas. Una reacción feminista frente a la nueva misoginia.

Whitehead, M. (1991). Los conceptos y principios de la equidad en la salud. OPS/OMS, Washington, D.C.

Zambrano R., Castro D., Lozano M., Gómez, N., y Rojas, C. (2013). Conocimientos sobre VIH y comportamientos en Salud Sexual y Reproductiva en una comunidad indígena de Antioquia. Investigaciones Andina, 15 (26), 640-652. 